Aim of the study: Despite recent technical progress and advances in the perioperative management of liver surgery, postoperative surgical site infection (SSI) is still one of the most common complications that extends hospital stays and increases medical expenses following hepatic surgery. Material and methods: From 2001 to 2017 a total of 1180 patients who underwent hepatic resection for liver tumours were retrospectively analysed with respect to the predictive factor of superficial incisional SSI, using a propensity score matching by procedure (subcuticular or mattress suture).

Results: The incidence of superficial and deep incisional SSIs was found to be $7.1 \%(84 / 1180)$. By propensity score matching (PSM), 121 of the 577 subcuticular suture group patients could be matched with 121 of the 603 mattress suture group patients. Multivariate analysis demonstrated wound closure technique as the only independent risk factor that correlated significantly with the occurrence of superficial incisional SSIs $(p=0.038)$ C-reactive protein (CRP) levels on postoperative day 4 were significantly higher in patients with incisional SSIs than in those without $(p<0.001)$.

Conclusions: Wound closure technique with subcuticular continuous spiral suture using absorbable suture should be considered to minimise the incidence of incisional SSIs. Moreover, wounds should be carefully checked when CRP levels are high on postoperative day 4.

Key words: superficial incisional SSI, hepatic resection, subcuticular suture, mattress suture.

Contemp Oncol (Pozn) 2018; 22 (3): 184-190 DOI: https://doi.org/10.5114/wo.2018.78940

\section{The utility of the subcuticular suture in hepatic resection}

\author{
Yoshihiro Inoue, Kensuke Fujii, Masatsugu Ishii, Syuji Kagota, \\ Hiroki Hamamoto, Wataru Osumi, Yusuke Tsuchimoto, Shinsuke Masubuchi, \\ Masashi Yamamoto, Akira Asai, Koji Komeda, Shinya Fukunishi, \\ Fumitoshi Hirokawa, Kazuhide Higuchi, Kazuhisa Uchiyama
}

Osaka Medical College Hospital, Takatsuki, Osaka, Japan

\section{Introduction}

Hepatic resection has played a leading role in the treatment of malignant liver tumours, including hepatocellular carcinoma (HCC) and liver metastases from colorectal carcinoma (CRCLM). However, hepatic resection is an invasive operation that can cause severe physiological stress responses and postoperative complications, such as surgical site infection (SSI), bile leakage, or liver dysfunction. Despite recent technical progress [1, 2] and advances in perioperative management of liver surgery, postoperative infection is still one of the most common complications, with a reported incidence of about 2.1-14.5\% [3-5]. Interestingly, the incidence of this complication has not changed much during the past decade, despite decreased morbidity and mortality rates after hepatic resection [6-8]. The prevention of SSIs is very important because they extend hospital stays and increase medical expenses following hepatic surgery. However, an SSI is a postoperative complication that occurs frequently after gastrointestinal surgery, including hepato-biliary surgery.

To the best of our knowledge, some studies have focused on identifying risk factors for incisional SSIs in superficial and deep regions of the abdominal wall after hepatic resection [9-11]. Although some risk factors for incisional SSIs have been identified, the relevance of these factors varies among studies.

The purpose of this retrospective study was to clarify the importance of wound closure technique as one of the perioperative risk factors for postoperative incisional SSI after elective hepatic resection in patients with liver tumours at a single institution.

\section{Material and methods}

Patient population and selection

From January 17, 2001 to December 18, 2017 a total of 1180 patients underwent curative open hepatic resection for liver tumours at Osaka Medical College Hospital in Takatsuki City, Japan. All patients were fully informed of the study design according to the Ethics Committee on Clinical Investigation of Osaka Medical College Hospital (No. 1029) and provided written, informed consent. Patients with an associated biliary-enteric anastomosis and gastrointestinal procedures were excluded from the study. All patient characteristics had been collected prospectively in a database. Missing data were obtained by reviewing patient files.

The preoperative workup consisted of a specified protocol, including blood examinations, abdominal ultrasound, angio-computed tomography (CT) scan, and magnetic resonance imaging (MRI). Evaluation of hepatic function was performed using the Child-Pugh classification [12] of liver dysfunction. Patients with complicated cirrhosis (Child-Pugh class C) or an American Society of Anaesthesiology (ASA) classification greater than 4 would not be appropriate for liver resection. 
Demographic and clinical variables were recorded on admission. The data for the 368 hepatic resection patients included age, sex, body mass index (BMI), disease, viral infection status, history of previous operations, type of operation, and administration of perioperative antibiotics. Serum total bilirubin, albumin, aspartate aminotransferase (AST), alanine aminotransferase (ALT), platelet count, prothrombin time (PT), and indocyanine green retention rate at 15 min (ICG-R15) were measured at the Central Laboratory of our hospital.

\section{Surgical and pathological factors}

The surgical factors assessed included surgical duration, intraoperative blood loss, blood transfusion requirement, repeat operation, operative method, type and method of hepatic resection, abdominal drain insertion, and wound closure technique. Pathological factors evaluated intra- and postoperatively included number of tumours, size of the largest tumour, and background liver histology.

\section{Surgical procedure}

The indications for surgical resection were in accordance with the criteria of Makuuchi et al. [13]. Details of the surgical technique routinely used in our department have been described in previous reports [14-16]. The rectus sheath was closed with synthetic absorbable braided sutures (Vicryl No. 1; Ethicon, Tokyo, Japan). The skin was irrigated with approximately $500 \mathrm{ml}$ of warm saline. From 2001 to 2011, skin was stitched with vertical mattress sutures using 2-0 monofilament non-absorbable suture (polyamide; Nylon, Ethicon, Tokyo, Japan). From 2012 to 2017 skin was stitched with subcuticular continuous spiral stitches using 4-0 monofilament absorbable suture (polydioxanone; PDS-II, Ethicon, Tokyo, Japan). The wound was covered for $72 \mathrm{~h}$ postoperatively by Opsite Post-Op Visible (Smith \& Nephew Medical Limited, Tokyo, Japan).

Antibiotics were injected intravenously (IV) within 30 min prior to skin incision. In patients who underwent operations that lasted longer than three hours, additional antimicrobial agents were intravenously injected every three hours, as recommended by the Centres for Disease Control and Prevention (CDC) guidelines [17]. Postoperative antibiotics were administered for 48 hours intravenously. Appropriate oral intake was resumed on postoperative day 2 . All patients were followed for at least 30 days after surgery to monitor for the development of SSIs or other postoperative complications.

\section{Definitions}

SSIs were defined according to the CDC's National Nosocomial Infection Surveillance (NNIS) system [17]. Using these criteria, SSIs are classified as being either incisional (superficial or deep) or organ/space. Criteria for a superficial incisional SSI included an infection occurring at the incision site within 30 days after surgery, which involved only the skin and subcutaneous tissue and at least one of the following: pus discharge from the incision; bacteria isolated from a sample culture from the superficial incision; localised pain, tenderness, swelling, redness, or heat; or wound dehiscence. Criteria for a deep incisional SSI included an infection of the fascia or muscle related to the surgical pro- cedure occurring within 30 days of surgery and at least one of the following: pus drainage from the deep incision; spontaneous dehiscence of the incision; or deliberate opening of the incision when the patient had the previously described signs and symptoms of infection. Each SSI was diagnosed and confirmed by a surgeon other than the patient's primary surgeon or by the institution's infection control team.

Complications were classified according to the Clavien-Dindo classification of surgical complications [18, 19]. All patients who underwent hepatic resection were monitored for the assessment of pain. A visual analogue scale (VAS; 0-100) and analgesic use were used for pain assessment $1,2,4$, and 7 days after surgery.

Operative procedures were classified according to conventional terminology derived from the eight segments of the liver, as suggested by Couinaud [20]. Anatomic resection was defined as resection of the neoplasm together with the portal vein related to the neoplasm and the corresponding hepatic territory. Non-anatomic resection was defined as resection of a lesion without regard to segmental, sectional, or lobar anatomy.

\section{Statistical analysis}

To minimise the influence of potential confounders on selection bias, propensity scores were generated by using binary logistic regression. Independent variables entered into the propensity model included preoperative information. One-to-one matching between groups was accomplished by using the nearest-neighbour matching method. This matching was performed without replacement and by using a calliper width of 0.2 standard deviations of the logit of the estimated propensity score. After propensity score matching analysis (PSM), the two matched groups were handled as unpaired independent groups. Continuous variables were expressed as medians \pm standard deviation. Univariate analyses were compared by the Student's t-test and $\chi^{2}$ test, Mann-Whitney's $U$ test, Wilcoxon signed-rank test, or Fisher's exact test, as appropriate. Multivariate analyses were performed by Cox proportional hazards regression. Values of $p<0.05$ were considered significant. All statistical analyses were performed using JMP version 12 .

\section{Results}

\section{Background data of patients who underwent hepatic resection}

Data from 1180 hepatic resection patients $(777$ men, 403 women; median age 68 years, range 0-89 years) without biliary-enteric anastomoses and gastrointestinal procedures were analysed. Patient demographics, tumour characteristics, the type of hepatic resection performed, intraoperative factors, and short-term outcome following hepatic resection were summarised. The median BMI was 22.4 (range, $15.1-36.2) \mathrm{kg} / \mathrm{m}^{2}$. The predominant cause for chronic liver disease was related to chronic hepatitis B or C infection, and 535 (45.3\%) patients were found to have positive serology for hepatitis B surface antigen or hepatitis C virus (HCV) antibody. A total of 582 patients had HCC (49.3\%), 405 patients had metastatic liver tumours from colorectal cancer (34.3\%), and 193 patients had other liver tumours (16.4\%). 
Table 1. Clinical and laboratory data of patients who underwent hepatic resections before propensity score matching and after propensity score matching

\begin{tabular}{|c|c|c|c|}
\hline Characteristic & Subcuticular suture & Mattress suture & $p$-value \\
\hline Before PSM & $n=577$ & $n=603$ & \\
\hline Age, years, median (range) & $69(0-89)$ & $67(21-89)$ & $0.002^{*}$ \\
\hline Gender, male, $n$ (\%) & $371(64.3)$ & $406(67.3)$ & 0.255 \\
\hline BMI, kg/m², median (range) & $22.6(11.9-34.9)$ & $22.2(14.9-36.2)$ & 0.257 \\
\hline Smoking, $n(\%)$ & $308(53.5)$ & $263(43.6)$ & $0.014^{*}$ \\
\hline Hepatitis viral infection, $n(\%)$ & $234(40.6)$ & 301 (49.9) & $0.001^{\star}$ \\
\hline Diabetes mellitus, $n$ (\%) & $154(26.7)$ & $118(19.6)$ & $0.004^{*}$ \\
\hline HCC/Metastasis, others & $256 / 321$ & $326 / 277$ & $0.001^{\star}$ \\
\hline Albumin, g/dl, median (range) & $4.0(2.0-5.2)$ & $4.0(1.8-5.2)$ & $0.004^{*}$ \\
\hline Platelet count, $\times 10^{4} / \mu l$, median (range) & $19.0(1.9-76.5)$ & $19.3(2.9-69.1)$ & 0.340 \\
\hline Child-Pugh classification, A, $n$ (\%) & $560(97.1)$ & $543(90.0)$ & $<0.001^{*}$ \\
\hline Number of tumours & $1(1-24)$ & $1(1-13)$ & $0.010^{*}$ \\
\hline Size of largest tumour (cm) & $2.8(0.5-18.9)$ & $3.2(0.8-21.0)$ & 0.161 \\
\hline Repeat operation, $n$ & $123(21.3)$ & $72(11.9)$ & $<0.001^{\star}$ \\
\hline $\begin{array}{l}\text { Type of hepatic resection, } n \text { (\%) } \\
\text { Lobectomy } \\
\text { Segmentectomy } \\
\text { Partial resection }\end{array}$ & $\begin{array}{l}101(17.5) \\
108(18.7) \\
368(63.8)\end{array}$ & $\begin{array}{l}167(27.7) \\
150(24.9) \\
286(47.4)\end{array}$ & $<0.001^{\star}$ \\
\hline Operative time (min) & $208(30-980)$ & $335(50-1510)$ & $<0.001^{\star}$ \\
\hline Blood loss (ml) & $210(0-10970)$ & $468(0-21770)$ & $<0.001^{\star}$ \\
\hline Blood transfusion (\%) & $134(23.2)$ & $140(23.2)$ & 0.977 \\
\hline Intraoperative abdominal drainage (\%) & $235(40.8)$ & $332(55.1)$ & $<0.001^{\star}$ \\
\hline Duration of antibiotics (day) & $3(1-14)$ & $2(1-13)$ & $<0.001^{\star}$ \\
\hline After PSM & $n=121$ & $n=121$ & \\
\hline Age, years, median (range) & $69(28-85)$ & $69(29-89)$ & 0.790 \\
\hline Gender, male, $n$ (\%) & $81(66.9)$ & $78(64.5)$ & 0.685 \\
\hline BMI, kg/m², median (range) & $22.3(13.9-32.9)$ & $22.2(16.9-36.2)$ & 0.657 \\
\hline Smoking, $n(\%)$ & $66(54.6)$ & $55(45.5)$ & 0.157 \\
\hline Hepatitis viral infection, $n(\%)$ & $59(48.8)$ & $61(50.4)$ & 0.898 \\
\hline Diabetes mellitus, $n$ (\%) & $29(24.0)$ & $27(22.3)$ & 0.761 \\
\hline HCC/Metastasis, others & $66 / 55$ & $68 / 53$ & 0.796 \\
\hline albumin, g/dl, median (range) & $4.0(2.9-5.2)$ & $4.0(1.8-4.9)$ & 0.933 \\
\hline Platelet count, $\times 10^{4} / \mu \mathrm{l}$, median (range) & $19.3(2.6-42.2)$ & $18.2(3.5-45.5)$ & 0.258 \\
\hline Child-Pugh classification, A, $n$ (\%) & $118(97.5 \%)$ & $115(95.0)$ & 0.308 \\
\hline Number of tumours & $1(1-10)$ & $1(1-12)$ & 0.602 \\
\hline Size of largest tumour (cm) & $2.8(0.6-12.5)$ & $2.8(0.8-13.0)$ & 0.424 \\
\hline Repeat operation, $n$ (\%) & $21(17.4 \%)$ & $22(18.2)$ & 0.867 \\
\hline $\begin{array}{l}\text { Type of hepatic resection, } n \text { (\%) } \\
\text { Lobectomy } \\
\text { Segmentectomy } \\
\text { Partial resection }\end{array}$ & $\begin{array}{l}15(12.4) \\
30(24.8) \\
76(62.8)\end{array}$ & $\begin{array}{c}9(7.4) \\
30(24.8) \\
82(67.8)\end{array}$ & 0.422 \\
\hline Operative time (min) & $254(38-666)$ & $275(50-505)$ & 0.511 \\
\hline Blood loss (ml) & $230(0-9340)$ & $330(0-2915)$ & 0.993 \\
\hline Blood transfusion, $n(\%)$ & $22(18.2)$ & $24(19.8)$ & 0.870 \\
\hline Intraoperative abdominal drainage, $n$ (\%) & $51(42.2)$ & $50(41.3)$ & 0.896 \\
\hline Duration of antibiotics (day) & $1(1-7)$ & $2(1-13)$ & 0.659 \\
\hline
\end{tabular}

$p<0.05$; BMI - body mass index; HCC - hepatocellular carcinoma; PSM - propensity score matching 
Patients were enrolled between January 2001 and December 2017 and divided into two groups: subcuticular suture group $(n=577)$ and mattress suture group $(n=603)$ (Table 1$)$. HCC and Child-Pugh classification B were more common on histology and laboratory data in the mattress suture group than in the subcuticular suture group $(p=0.001$ and $<0.001)$. The operative time, blood loss, intraoperative abdominal drainage, and duration of antibiotics were more frequent and thus longer in the subcuticular suture group $(p<0.001$, $<0.001,<0.001$, and $<0.001$, respectively). The VAS scores at 1, 2, 4, and 7 days after surgery were $42,37,20$, and 3 , and differences between groups were not significant $(p=0.886$, $0.261,0.438$, and 0.362 , respectively). There was also no significant difference in the use of additional analgesia between groups ( $p=0.542,0.984,0.158$, and 0.156 , respectively).

The median follow-up period of all patients was 21 months (range, 0-194 months). Overall, superficial, and deep incisional SSIs after hepatic resection occurred in 84 of 1180 patients (7.1\%), and 1096 (92.9\%) patients had no SSIs after hepatic resection. A superficial incisional SSI was found in 84 patients (7.1\%), and a deep incisional SSI was found in 18 patients (1.5\%).

\section{Propensity score matching}

By propensity score matching (PSM), 121 of the 577 subcuticular suture group patients could be matched with 121 of the 603 mattress suture group patients. The baseline characteristics of the matched study population (242 patients) are summarised in Table 1 . There were no significant differences in the demographic or operative characteristics between the groups. The number and size of the tumours were similar between the groups, which could be treated by either subcuticular suture or mattress suture.

\section{Incidence of superficial and deep incisional SSIs after hepatic resection}

The subcuticular suture group had a $22.4 \%$ complication rate, while the mattress suture group had a complication rate of $23.7 \%$, although this difference was not significant $(p=0.847)$. Early-stage complications following surgical treatment, including the incidences of SSIs within 30 days of surgery, are compared in Table 2. For patients with subcuticular suture or mattress suture, hospital mortality rates were $2.5 \%$ and $0.8 \%$, respectively $(p=0.313)$. The incidence of superficial incisional SSIs was significantly lower in the subcuticular suture group (3.3\%) than in the mattress suture group $(10.7 \%)(p=0.024)$. The incidence of deep incisional and space/organ SSIs was no different between the two groups ( $p=0.317$ and 0.734 , respectively). Bacterial cultures from superficial and deep incisional SSIs in the subcuticular suture group identified Enterococcus spp. in two cases, Staphylococcus spp. in one, and Streptococcus spp. in one. Cultures in the mattress suture group identified Staphylococcus spp. in eight cases,

Table 2. Surgical outcome of patients who underwent hepatic resections after propensity score matching

\begin{tabular}{|c|c|c|c|}
\hline Surgical outcome & $\begin{array}{l}\text { Subcuticular suture } \\
\qquad(n=121)\end{array}$ & $\begin{array}{l}\text { Mattress suture } \\
\quad(n=121)\end{array}$ & $p$-value \\
\hline Postoperative complications, $n$ (\%) & $17(22.4)$ & $18(23.7)$ & 0.847 \\
\hline Mortality, n (\%) & $3(2.5)$ & $1(0.8)$ & 0.313 \\
\hline Superficial incisional SSIs, $n$ (\%) & $4(3.3)$ & $13(10.7)$ & $0.024^{\star}$ \\
\hline Deep incisional SSIs, $n$ (\%) & $3(2.5)$ & $1(0.8)$ & 0.317 \\
\hline Organ/space SSIs, $n(\%)$ & $5(4.2)$ & $4(3.3)$ & 0.734 \\
\hline Postoperative hospital stay (day) & $12(5-124)$ & $14(5-172)$ & 0.467 \\
\hline
\end{tabular}

Table 3. Bacterial culture from superficial and deep incisional surgical site infections and antibiotic sensitivity

\begin{tabular}{|c|c|c|c|c|c|c|c|c|}
\hline Bacterial culture & Number & $A B P C$ & $\mathrm{CMZ}$ & IPM & MINO & CLDM & LVFX & VCM \\
\hline $\begin{array}{l}\text { Staphylococcus spp. } \\
\text { Staphylococcus aureus } \\
\text { CNS }\end{array}$ & $\begin{array}{l}2 \\
5\end{array}$ & & $\begin{array}{l}S \\
R\end{array}$ & $\begin{array}{l}S \\
R\end{array}$ & $\begin{array}{l}S \\
S\end{array}$ & $\begin{array}{l}S \\
S\end{array}$ & $\begin{array}{l}S \\
S\end{array}$ & $\begin{array}{l}S \\
S\end{array}$ \\
\hline MSSA & 2 & $S$ & $S$ & $S$ & $S$ & $S$ & S & $S$ \\
\hline MRSA & 1 & & $\mathrm{R}$ & $\mathrm{R}$ & $S$ & $S$ & S & $S$ \\
\hline Streptococcus spp. & 1 & $\mathrm{~S}$ & & $S$ & & $S$ & $S$ & $S$ \\
\hline $\begin{array}{l}\text { Enterococcus spp. } \\
\text { Enterococcus faecalis } \\
\text { Enterococcus faecium }\end{array}$ & $\begin{array}{l}2 \\
2\end{array}$ & $\begin{array}{l}S \\
R\end{array}$ & & $\begin{array}{l}S \\
R\end{array}$ & $\begin{array}{l}S \\
R\end{array}$ & & $\begin{array}{l}S \\
R\end{array}$ & $\begin{array}{l}S \\
S\end{array}$ \\
\hline Enterobacter cloacae & 1 & $\mathrm{R}$ & $R$ & $S$ & $S$ & & $S$ & \\
\hline Pseudomonas aeruginosa & 1 & & & S & & & S & S \\
\hline
\end{tabular}

CNS - coagulase-negative staphylococci; MSSA - methicillin-sensitive staphylococcus aureus; MRSA - methicillin-resistant staphylococcus aureus; ABPC ampicillin; CMZ - cefmetazole; IPM - imipenem; MINO - minocycline; CLDM - clindamycin; LVFX - levofloxacin; VCM - vancomycin; S-sensitive; R-resistant 
Table 4. Predictors of postoperative superficial incisional surgical site infections: multivariate analysis

$\begin{array}{lccc}\text { Factor } & \text { p-value } & \text { OR } & \text { 95\% Cl } \\ \text { Wound closure technique (mattress) } & 0.038^{*} & 3.157 & 1.060-11.582 \\ \text { Operative time } \geq 300 \text { (min) } & 0.267 & 1.794 & 0.631-5.097 \\ { }^{*} p<0.05 ; \text { OR - odds ratio; } \mathrm{Cl}-\text { confidence interval } & & \end{array}$

Enterococcus spp. in two, Enterobacter spp. in one, Streptococcus spp. in one, methicillin-resistant $S$. aureus in one, and Pseudomonas aeruginosa in one. Normal skin bacteria were found in $10(58.8 \%)$ of 17 cases (Table 3$)$.

In patients with subcuticular suture, the median duration of postoperative hospital stay was 12 days (range, 5-124 days), in comparison with patients with mattress suture, in whom it was 14 days (range, 5-172 days). Thus, postoperative hospital stay was no different between the two groups ( $p=0.467)$. However, the median duration of postoperative hospital stay was 27 days (range, 14-172 days) for postoperative incisional SSI cases, and 13 days (range, 5-124 days) for cases without a postoperative incisional SSI $(p<0.001)$. The subcuticular suture group had a mortality rate of $2.5 \%$, while the $\mathrm{OH}$ group had a mortality rate of $0.8 \%$, although this difference was not significant ( $p=0.313)$. These four patients $(1.7 \%)$ died due to posthepatectomy liver failure without incisional SSIs.

\section{Risk factors for postoperative superficial} incisional SSIs after hepatic resection

Perioperative factors were compared between patients with and without postoperative superficial incisional SSIs. Thirty-five factors were examined, including patient factors, tumour factors, and operative factors.

Of the factors analysed, operative time $(p=0.017)$ and wound closure technique ( $p=0.039$ ) were found to be significant risk factors for postoperative incisional SSIs.

The duration of surgery was significantly longer in patients with incisional SSIs than in those without $(298 \pm 133$ vs. $256 \pm 113 \mathrm{~min}$, respectively; $p=0.022$ ). Receiver-operator characteristic (ROC) curve analysis indicated that the optimal cut-off value for operative time was 295.0 min, yielding $87.5 \%$ sensitivity and $66.1 \%$ specificity for the occurrence of incisional SSIs.

Multivariate analysis demonstrated that wound closure with interrupted stitches using nylon ( $p=0.038$; odds ratio [OR] 3.157; 95\% confidence interval [Cl] 1.060-11.582) and operative time $\geq 300 \mathrm{~min}(p=0.267$; OR 1.794; $95 \% \mathrm{Cl}$ 0.631-5.097) were independent risk factors for the occurrence of postoperative incisional SSIs (Table 4).

Postoperative laboratory data with superficial and deep incisional SSIs after hepatic resection

Postoperative serum AST and ALT levels peaked on day 1 and had almost normalised by day 7 . Although serum total bilirubin and prothrombin time peaked on day 1 , they remained within the normal ranges in both groups. Postoperative serum white blood cell (WBC) counts (Fig. 1A) and C-reactive protein (CRP) levels (Fig. 1B) peaked on day 2 and then gradually normalised. However, CRP levels of patients with incisional SSIs, in all postoperative courses after postoperative day 4, were significantly higher than those of patients without incisional SSIs. CRP levels on postoperative day 4 were significantly higher in patients with incisional SSIs than in those without $(9.7 \pm 5.6$ vs. $6.7 \pm 0.4 \mathrm{mg} / \mathrm{dl}$, respectively; $p<0.001)$. ROC curve analysis indicated that the optimal cutoff for CRP levels was $15.0 \mathrm{mg} / \mathrm{dl}$, yielding 41.7\% sensitivity and $94.0 \%$ specificity for the occurrence of incisional SSIs.

\section{Discussion}

In the surgery, the result of wound suture is one of the most important results. All surgeons want cosmetically acceptable wound scars along with optimal healing, and all patients also expect similar results. However, the incidence of SSIs following surgical treatment for liver cancer is rela-
A

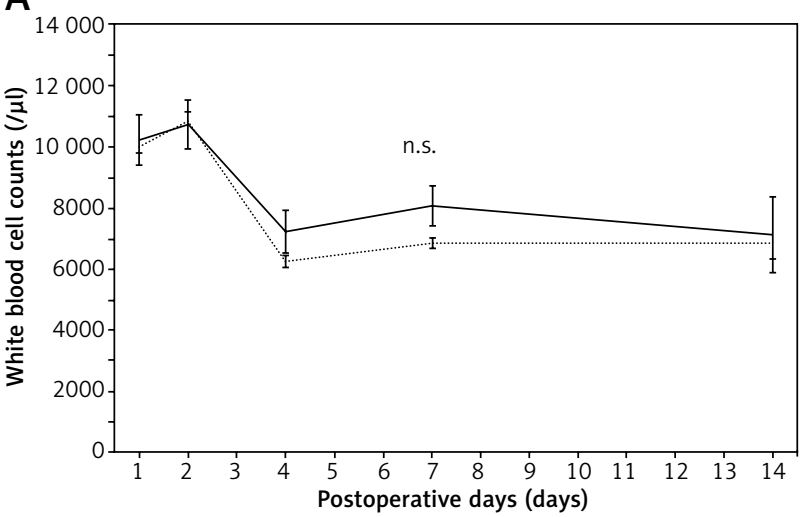

B

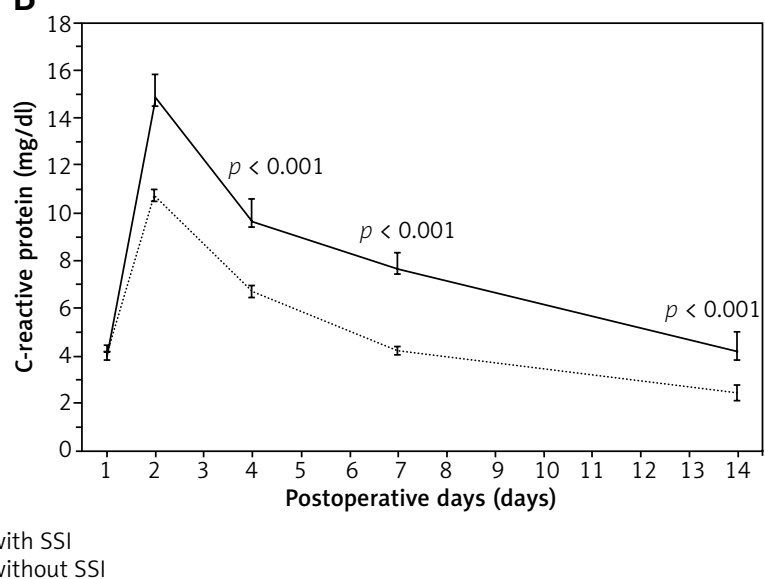

Fig. 1. Postoperative changes in white blood cell counts and C-reactive protein levels with and without superficial and deep incisional surgical site infections (means \pm standard error) A) White blood cell counts $(/ \mu \mathrm{l})$. B) C-reactive protein levels (mg/dl) 
tively high. An incisional SSI is not a critical complication by itself, but the occurrence of an SSI can prolong postoperative hospital stays and increase medical expenses.

Therefore, this study retrospectively compared the effectiveness of subcuticular suture and mattress suture in hepatic resections from the perspective of the short-term outcome at a single institution. This comparative study using PSM analysis, although retrospective, confirmed that the subcuticular suture is an effective therapeutic option with a lower incidence rate of superficial incisional SSIs than that of the mattress suture. In this study, the patient characteristics of the subcuticular and mattress suture groups were quite similar by PSM analyses even though it was not a randomised, controlled trial and the number of patients was small. Although the small number of patients in each arm of our study and the retrospective nature of the control group makes the chance of a statistical error possible, the conclusions seem reliable. The potential historical bias is reduced by the design of the study resulting in the subcuticular suture group that was well matched with the mattress suture group for all demographic data. The preoperative management of patients was also similar in the two groups.

In the present study, multivariate analysis showed that wound closure technique was associated with postoperative superficial incisional SSIs. Interestingly, there was no preoperative laboratory factor, and only one operative factor found to be significantly associated with superficial incisional SSIs. Operative time was also associated in univariate analysis. Some investigators have addressed the relationship between operative time and incisional SSIs in a wide range of surgical procedures in general surgery [21, 22]. Surgical stress, such as prolonged operative time, impairs remnant liver function. Postoperative liver dysfunction may cause immune dysfunction and affect the development of incisional SSIs. A long operation also exposes the incision to desiccation, which may increase the likelihood of incisional contamination.

The only risk factor observed in the present multivariate analysis was wound closure technique. Traditionally, the suture material used commonly in wound closure was a vertical mattress suture using non-absorbable nylon because of its non-infection, inertness, and ease of use [23]. Recently, subcuticular suture has been reported to be highly effective for preventing incisional SSIs, especially in gynaecological surgery, without the need to remove the stiches [24].

For normal wound healing, there are various important factors. First, appropriate wound suture can join each layer of the separated wound edges, support reconstruction, and promote normal wound healing. However, inappropriate suture results in introversion of the wound edge and dead space between the separated wound edges. Fluid collection in the dead space may progress to a focus of wound infection and develop superficial and deep incisional SSIs. Moreover, the infection barrier of the epidermis is broken by wound sutures through the epidermis from outside, such as mattress sutures and staples. The bacteria may pass through the needle hole from outside and contaminate the wound. Therefore, subcuticular suture is theoretically more appropriate than mattress suture. In fact, normal skin bacteria accounted for more than half of the isolates from infected wounds in this study. Therefore, reducing contamination by these bacteria is important. Second, the blood circulation of the epidermis plays important roles. Perforating branches of the epidermis run horizontally. When the separated wound edges are sutured, these perforating branches should be preserved to prevent tissue ischaemia. Therefore, subcuticular suture should performed vertically or spirally. Finally, in regards to whether subcuticular suture should be interrupted or continuous, there are few recent articles describing subcuticular suture in detail, and this remains controversial [25]. However, the continuous method of closure has some advantages, namely quick closure with a smaller number of knots, thereby lessening the chances of sinus formation. Therefore, since 2012 we have closed with a subcuticular spiral continuous suture procedure using 4-0 monofilament absorbable suture. We found that wound closure technique with subcuticular continuous spiral suture using 4-0 monofilament absorbable suture was more effective than interrupted mattress suture using 2-0 monofilament nonabsorbable suture.

Incisional SSIs were also evaluated using the postoperative laboratory data because they are related to the postoperative clinical course. Postoperative laboratory data are believed to predict postoperative morbidity by quantifying the patient's inflammatory response. Only CRP levels after postoperative day 4 were found to be significantly associated with the occurrence of superficial and deep incisional SSIs. CRP levels were associated in a subgroup analysis likewise. In other words, incisional SSIs should be carefully checked when CRP levels on postoperative day 4 are $>15.0 \mathrm{mg} / \mathrm{dl}$. The wound was covered for three postoperative days and uncovered on postoperative day 4 . The wound was then checked for evidence of infection, such as pus discharge from the incision, localised pain, tenderness, swelling, or redness.

\section{Conclusions}

Wound closure technique with subcuticular continuous spiral suture using absorbable suture should be considered to minimise the incidence of superficial incisional SSIs following surgery for malignant liver tumours. Moreover, the wound should be carefully checked when CRP levels are high on postoperative day 4.

\section{The authors declare no conflicts of interest.}

\section{References}

1. Uchiyama K, Ueno M, Ozawa S, et al. Combined intraoperative use of contrast-enhanced ultrasonography imaging using a sonazoid and fluorescence navigation system with indocyanine green during anatomical hepatectomy. Langenbecks Arch Surg 2011; 396: 1101-1107

2. Uchiyama K, Ueno M, Ozawa S, Kiriyama S, Shigekawa Y, Yamaue $H$. Combined use of contrast-enhanced intraoperative ultrasonography and a fluorescence navigation system for identifying hepatic metastases. World J Surg 2010; 34: 2953-2959. 
3. Uchiyama K, Ueno M, Ozawa S, Kiriyama S, Kawai M, Hirono S, Tani M, Yamaue H. Risk factors for postoperative infectious complications after hepatectomy. J Hepatobiliary Pancreat Sci 2011; 18: 67-73.

4. Okabayashi T, Nishimori I, Yamashita K, Sugimoto T, Yatabe T, Maeda H, Kobayashi M, Hanazaki K. Risk factors and predictors for surgical site infection after hepatic resection. J Hosp Infect 2009; 73: 47-53.

5. Kobayashi S, Gotohda N, Nakagohri T, Takahashi S, Konishi M, Kinoshita T. Risk factors of surgical site infection after hepatectomy for liver cancers. World J Surg 2009; 33: 312-317.

6. Imamura H, Seyama Y, Kokudo N, Maema A, Sugawara Y, Sano K, Takayama T, Makuuchi M. One thousand fifty-six hepatectomies without mortality in 8 years. Arch Surg 2003; 138: 1198-1206.

7. Jarnagin WR, Gonen M, Fong Y, et al. Improvement in perioperative outcome after hepatic resection: analysis of 1803 consecutive cases over the past decade. Ann Surg 2002; 236: 397-406.

8. Poon RT, Fan ST, Lo CM, Liu CL, Lam CM, Yuen WK, Yeung C, Wong J. Improving perioperative outcome expands the role of hepatectomy in management of benign and malignant hepatobiliary diseases: analysis of 1222 consecutive patients from a prospective database. Ann Surg 2004; 240: 698-708.

9. Togo S, Matsuo K, Tanaka K, et al. Perioperative infection control and its effectiveness in hepatectomy patients. J Gastroenterol Hepatol 2007; 22: 1942-1948.

10. Nanashima A, Arai J, Oyama S, et al. Associated factors with sur gical site infections after hepatectomy: Predictions and countermeasures by a retrospective cohort study. Int J Surg 2014; 12: 310 314.

11. Moreno Elola-Olaso A, Davenport DL, Hundley JC, Daily MF, Gedaly R. Predictors of surgical site infection after liver resection: a multicentre analysis using National Surgical Quality Improvement Program data. HPB (Oxford) 2012; 14: 136-141.

12. Pugh RN, Murray-Lyon IM, Dawson JL, Pietroni MC, Williams R. Transection of the oesophagus for bleeding oesophageal varices. Br J Surg 1973; 60: 646-649.

13. Makuuchi M, Kosuge T, Takayama T, Yamazaki S, Kakazu T, Miyagawa S, Kawasaki S. Surgery for small liver cancers. Semin Surg Oncol 1993; 9: 298-304

14. Inoue $\mathrm{Y}$, Hayashi $\mathrm{M}$, Komeda $\mathrm{K}$, et al. Resection margin with anatomic or nonanatomic hepatectomy for liver metastasis from colorectal cancer. J Gastrointest Surg 2012; 16: 1171-1180.

15. Inoue Y, Hayashi M, Tanaka R, Komeda K, Hirokawa F, Uchiyama K. Short-term results of laparoscopic versus open liver resection for liver metastasis from colorectal cancer: a comparative study. Am Surg 2013; 79: 495-501.

16. Inoue Y, Tanaka R, Komeda K, Hirokawa F, Hayashi M, Uchiyama K. Fluorescence detection of malignant liver tumors using 5-aminolevulinic acid-mediated photodynamic diagnosis: principles, technique, and clinical experience. World J Surg 2014; 38: 1786-1794.

17. Mangram AJ, Horan TC, Pearson ML, Silver LC, Jarvis WR. Guideline for prevention of surgical site infection, 1999 Centers for Disease Control and Prevention (CDC) Hospital Infection Control Practices Advisory Committee. Am J Infect Control 1999; 27: 97-132.

18. Dindo D, Demartines N, Clavien PA. Classification of surgical com plications: a new proposal with evaluation in a cohort of $6336 \mathrm{pa}$ tients and results of a survey. Ann Surg 2004; 240: 205-213.

19. Clavien PA, Barkun J, de Oliveira ML, et al. The Clavien-Dindo classification of surgical complications: five-year experience. Ann Surg 2009; 50: 187-196.

20. Couinaud C. Surgical anatomy of the liver. Several new aspects. Chirurgie 1986; 112: 337-342.

21. Haridas M, Malangoni MA. Predictive factors for surgical site infection in general surgery. Surgery 2008; 144: 496-501; discussion: 501-503.

22. Campbell DA Jr, Henderson WG, Englesbe MJ, et al. Surgical site infection prevention: the importance of operative duration and blood transfusion - results of the first American College of Surgeons - National Surgical Quality Improvement Program Best Practices Initiative. J Am Coll Surg 2008; 207: 810-820.

23. Moy RL, Lee A, Zalka A. Commonly used suture materials in skin surgery. Am Fam Physician 1991; 44: 2123-2128.
24. Ibrahim MI, Moustafa GF, Al-Hamid AS, Hussein MR. Superficial incisional surgical site infection rate after cesarean section in obese women: a randomized controlled trial of subcuticular versus in terrupted skin suturing. Arch Gynecol Obstet 2014; 289: 981-986.

25. Gurusamy KS, Toon CD, Allen VB, Davidson BR. Continuous versus interrupted skin sutures for non-obstetric surgery. Cochrane Database Syst Rev 2014; 14: CD010365.

\section{Address for correspondence}

\section{Yoshihiro Inoue}

Osaka Medical College Hospital

2-7 Daigakumachi

569-8686 Takatsuki, Osaka, Japan

e-mail: sur129@osaka-med.ac.jp

Submitted: 27.06 .2018

Accepted: $\quad 9.08 .2018$ 